\title{
Ultra-wide band energy harvesting for ultra-low power electronics applications
}

\author{
Nasr Rashid ${ }^{1}$, Mohamed Shehata ${ }^{2}$ \\ ${ }^{1}$ Department of Electrical Engineering, College of Engineering, Jouf University, Sakaka, Saudi Arabia \\ ${ }^{1}$ Department of Electrical Engineering, Faculty of Engineering, Al-Azhar University, Cairo, Egypt \\ ${ }^{2}$ SOURCE, Department of Research Training, Giza, Egypt
}

\begin{tabular}{l} 
Article Info \\
\hline Article history: \\
Received Jul 19, 2020 \\
Revised Sep 9, 2020 \\
Accepted Oct 1, 2020 \\
\hline
\end{tabular}

Keywords:

Energy harvesting

Impulse radio

Low-power electronics

Ultrawide band

\begin{abstract}
In this work, the feasibility of energy harvesting in the useful UWB band (i.e., 3.1-10.6 GHz) is analytically investigated. A typical UWB communications/EH chain in this band is modeled and analyzed, considering the spectral constraints imposed by the federal communications commission (FCC) to UWB signaling. Based on the developed model, accurate analytical expressions are derived for the average received powers of two common types of impulse radio UWB (IR-UWB) signaling waveforms. Numerical simulations on the system-level show excellent agreement with the obtained analytical expressions. Moreover, the DC power levels expected from spectrally constrained IR-UWB waveforms are extremely low (less than 0.3 microwatt) and, accordingly, provide useful guidelines for the design and development of ULP electronics applications in the sub-microwatt range.
\end{abstract}

This is an open access article under the CC BY-SA license.

\section{Corresponding Author:}

Nasr Rashid

Department of Electrical Engineering

College of Engineering, Jouf University

Sakaka, Saudi Arabia

Email: nasrrashid@ju.edu.sa

\section{INTRODUCTION}

The interests in energy harvesting $(\mathrm{EH})$ techniques have increased to enable wirelessly chargeable and self-powered electronic devices such as the internet of things (IoT) modules, radio frequency (RF) identification (RFID) tags and wireless sensor networks (WSN) [1]. Numerous efforts have been reported to demonstrate the feasibility of RF energy as a promising resource for EH applications, either on the system level (e.g., [2]), the device level (e.g., [3]), and/or the materials (e.g., [4]) of which these devices are fabricated. Most often, the harvested RF energy is collected either from single tone [5] or multi-tone communication signals [1], located at discrete parts of the spectrum. Since the emission frequencies of RF wireless communication systems are distributed over a sufficiently wide spectrum, the corresponding EH systems are expected to cover high bandwidths to collect the maximum possible RF energy [6]. Moreover, the frequencies of the RF signals incident to the input of an energy harvester are usually not predictable. Motivated by these technical requirements, there has been increasing interests in the design and development of ultra-wide band (UWB) devices/systems for EH. In [6], for instance, two rectifier designs have been reported and fabricated to operate from 0.6 to $3 \mathrm{GHz}$. In [7], two rectifier designs for UWB EH have been presented.

The designed rectifiers focus on $\mathrm{EH}$ in the frequency bands from 470 to $860 \mathrm{MHz}$, and between $250 \mathrm{MHz}$ and $3 \mathrm{GHz}$. More examples for the design of UWB energy harvesters are reported in [8, 9], where printed UWB microstrip antenna, based organic substrates, for RF EH applications from $2.4 \mathrm{GHz}$, up to more 
than $10 \mathrm{GHz}$. Such designs are very favorable for EH from different signals occupying different frequency bands of the spectrum, including UWB signals. From the afore-mentioned overview, it is clear that, the majority of the studies reported on the design and development of wide/narrow band energy harvesting rectennas have competed to demonstrate their individual capabilities in terms of the RF-to-DC conversion efficiency. However, according to [10], the RF-to-DC conversion efficiency of a rectenna is, in general, not only a function of the rectenna design, but also of its input waveform. Therefore, in another category of studies reported on RF EH, the signaling waveforms carrying this RF energy have been considered. For different RF waveforms having the same power level, the harvested DC power levels are strongly dependent on the particular RF signaling waveform and some of its time domain characteristics such as the highly desirable peak-to-average-power-ratio (PAPR) [11].

Fortunately, the interests in developing broad band energy harvesters to collect RF energy from high PAPR signals perfectly matches the temporal as well as the spectral characteristics of impulse radio-UWB (IR-UWB) waveforms. Despite the severe spectral constraints imposed by the Federal Communications Commission (FCC) to their spectra (less than $-41.3 \mathrm{dBm} / \mathrm{MHz}$ ), IR-UWB signals are allowed to occupy a bandwidth as large as $7.5 \mathrm{GHz}$ (i.e., $3.1 \mathrm{GHz}-10.6 \mathrm{GHz}$ ) [12], commonly called the useful UWB band. Moreover, in IR-UWB signaling, the energies of IR-UWB signals are emitted in the form of transient bursts of very short duration (typically, ns or ps) and large voltage amplitudes (compared to the DC levels of IRUWB waveforms). Meanwhile, due to the poor radiation efficiency of UWB antennas at low frequencies (below $1 \mathrm{GHz}$ ), the radiated IR-UWB signals often possess DC-null components [13]. This results in IRUWB signals with high PAPR levels. To the best of the authors' knowledge, no study has yet been reported to assess the maximum DC power levels that can be extracted from these signals. In this paper, a systemlevel abstraction approach is adopted to evaluate the maximum DC power levels expected from IR-UWB signals under the FCC spectral constraints, regardless of the specific realization of the EH circuit/system. Under this assumption, the calculated DC power levels are guaranteed to be the maximum achievable levels, regardless of the detailed architecture of the EH circuit and/or its RF-to-DC conversion efficiency.

The rest of this paper is organized as follows. A UWB communications/EH chain is described and is modeled in Section 2, considering typical IR-UWB signaling waveforms. In section 3, analytical expressions for the average DC powers harvested from the considered waveform types are developed. The developed expressions are then numerically evaluated and are analyzed in section 4 . Based on the obtained results, the whole work provided in this paper is finally concluded in section 5 .

\section{UWB SIGNALLING/ENERGY HARVESTING MODEL}

Figure 1 illustrates the block diagram of a typical IR-UWB signaling/EH chain, inspired from the models provided in [4]. Generally, it consists of an IR-UWB transmitter and an UWB energy harvester at the receiver side, each equipped with a broadband UWB antenna. At the transmitter side, an information source emits a stream of $M$-ary encoded symbols. Moreover, it is assumed that, the information source and its associated resistance, denoted by $R_{\mathrm{S}}$, model all signal processing functions that precede the TX antenna. Furthermore, the source resistance $R_{\mathrm{s}}$ is assumed to be matched to the input impedance of the TX antenna.

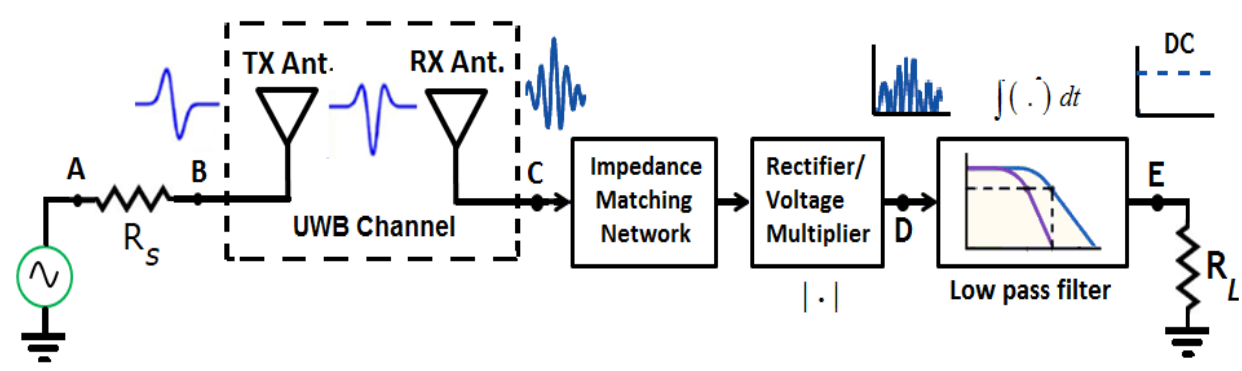

Figure 1. Block diagram of a typical IR-UWB communications/energy harvesting chain

Each of the emitted symbols is assumed to be simultaneously encoded by the pulse amplitude modulation (PAM) and the pulse position modulation (PPM) schemes. Each of the PAM/PPM modulated symbols is further encoded by an IR-UWB signaling waveform, denoted by $\phi(t)$, assigned to each symbol. The waveform stream at Point B in Figure 1 is expressed as follows: 


$$
x(t)=\sum_{j=-\infty}^{\infty} s_{j}^{q} \phi\left(t \pm \mu_{j}^{q}-j T_{s}\right)
$$

where $j$ is the time-domain symbol index, $s_{j}^{q}$ is the $q^{\text {th }}$ amplitude of the $j^{\text {th }}$ PAM encoded symbol, $q \in\{0,1 \ldots M-1\}$ is the index of the symbol alphabet, $M$ is the order of the modulation scheme, $T_{s}$ is the symbol duration and $\mu_{j}^{q}$ is $q^{\text {th }}$ time shift of the $j^{\text {th }}$ PPM modulated symbol. The \pm sign accounts for the two possibilities of time delay or time advance in PPM. It should be highlighted that, a probability of occurrence of $p_{q}$ is assigned to the $q^{\text {th }}$ information symbol. The spectrally shaped waveform stream is fed to an UWB Tx antenna before being radiated over an UWB wireless channel to a wirelessly located UWB Rx antenna. The impulse response of the UWB channel is based on the IEEE 802.15.3 standard model and is expressed as follows [14]:

$$
h(t)=\chi \sum_{l=1}^{L} \sum_{k=1}^{K} \alpha_{l, k} \beta_{l, k} \delta\left(t-T_{l}-\tau_{l, k}\right)
$$

where $\chi$ is a log-normal random variable that models the line-of-sight (LoS) path loss of the UWB channel, $L$ is the number of observed clusters, $K$ is the number of rays received within the $l^{\text {th }}$ cluster, $\alpha_{l, k}$ is the coefficient of the $k^{\text {th }}$ multipath contribution of $l^{\text {th }}$ cluster, $T_{l}$ is the time of arrival of the $l^{\text {th }}$ cluster, and $\tau_{l, k}$ the delay of the $k^{\text {th }}$ ray within the $l^{\text {th }}$ cluster, calculated with respect to $T_{l}$. The path loss variable $\chi$ is given by $\chi=G_{T} G_{R} c^{2} /\left(16 \pi^{2} D^{\gamma} f^{2}\right)$, where $G_{T}$ and $G_{R}$ are the gains of the Tx and the Rx UWB antennas, respectively, $\mathrm{c}=3 \times 10^{8} \mathrm{~m} / \mathrm{s}$ is the speed of light in free space, $D$ is the Tx-Rx antenna separation (in meters), $\gamma$ is the path loss exponent, and $f$ is the frequency at which the path loss is evaluated. It is important to highlight that $G_{T}$ and $G_{R}$ account for the radiation efficiency, polarization and the Ohmic losses exhibited by the antenna materials at the Tx and the Rx, respectively (Point B to Point $\mathrm{C}$ on Figure 1). The channel coefficient $a_{l, k}$ is a discrete random variable that assumes values of \pm 1 with equal probabilities, whereas $\beta_{l, k}$ is a Rayleigh distributed random variable that models the fading contribution of the $k^{\text {th }}$ ray in the $l^{\text {th }}$ cluster. The voltage waveform at the input of the Rx rectenna is given by:

$$
r(t)=x(t) * h(t)+n(t) \approx \sum_{j=-\infty}^{\infty} \sum_{p=1}^{P} w_{p} s_{j}^{q} \phi\left(t \pm \mu_{j}-j T_{s}-\tau_{p}\right)
$$

where $(*)$ is the linear convolution operator, $P=K L, w_{p}=\chi \alpha_{l, k} \beta_{l, k}, \tau_{p}=T_{l}+\tau_{l, k}$ is the overall ray delay and $n(t)$ is an additive white Gaussian noise (AWGN) process. In the following analysis, a high signalto-noise ratio (SNR) at the receiver front-end is assumed such that the AWGN term in (3) is negligible. This assumption is applicable to close proximity UWB systems where a high SNR is typically observed at the input of an UWB receiver. Moreover, a resolvable set of multipath components is assumed. This can be expressed mathematically as $T_{s} \gg \max \left\{\tau_{p}\right\}$ and $\tau_{p+1}-\tau_{p} \gg \tau ; p \in\{1,2 \ldots P\}$.

The received waveform is applied to the input of a matching network for maximum power transfer from the Rx antenna output to the input of a high speed full-wave rectifier (FWR)/voltage multiplier (VM). The output of the FWR/VM (Point D in Figure 1) is followed by a low-pass filter (LPF), such that the desired DC power of the received IR-UWB waveform is extracted at the LPF output. Without loss of generality, the FWR/VM applies the absolute value operator |.| to $r(t)$, whereas the LPF is modeled by an ideal timedomain integrator. The DC power obtained at the LPF output is delivered to a load resistance of $R_{L} W$; the Thevenin equivalent of the passive circuits and systems that follow the LPF. The load resistance is assumed to be matched to the output impedance of the rectenna configuration. Since a resolvable set of nonoverlapping multipath components is assumed, the extracted DC power of the received IR-UWB waveform is expressed as follows:

$$
\bar{P}_{D C}=\lim _{T \rightarrow \infty} \frac{1}{2 T R_{L}} \int_{-T}^{+T}|r(t)|^{2} d t=\sum_{q=0}^{M-1} \sum_{p=1}^{P}\left(\frac{p_{q} w_{p}^{2}\left|s_{j}^{q}\right|^{2}}{T_{s} R_{L}}\right) E_{\phi}
$$


where $E_{\phi}$ is defined as follows:

$$
E_{\phi} \triangleq \int_{-\infty}^{+\infty}\left|\phi\left(t \pm \mu_{j}-j T_{s}-\tau_{p}\right)\right|^{2} d t=\int_{-\infty}^{+\infty}|\phi(t)|^{2} d t
$$

is the DC energy of a single non-faded resolvable multipath component. From (4), it is clear that, due to the uncontrollable transmission loss, the harvested power $\bar{P}_{D C}$ can be evaluated and maximized with respect to the IR-UWB waveform and its associated parameters embedded in $E_{\phi}$. In this work, the IR-UWB signaling waveforms; $\phi(t)$ is assumed to be a time and/or amplitude scaled derivatives of a basis function, denoted by $\psi(t, \tau)$ where $\tau$ is its temporal pulse shaping factor. This basis function is either a typical amplitude-scaled Gaussian pulse which, expressed as $\psi(t, \tau)=\exp \left(-t^{2} / \tau_{g}^{2}\right)$ where $\tau_{g}$ is the Gaussian pulse width, or a typical amplitude-scaled hyperbolic secant (sech) pulse which is given by $\psi(t, \tau)=\operatorname{sech}\left(t / \tau_{s}\right)$ where $\tau_{s}$ is the sech pulse width. The pulse shaping factor of $\psi(t, \tau)$ is defined as its full width at half maximum (FWHM) pulse width and is related to the Gaussian and sech pulse widths as $\tau_{g} \triangleq \tau / 2 \sqrt{\log (2)}$ and $\tau_{s} \triangleq \tau / 2 \operatorname{sech}^{-1}(0.5)$ respectively. Accordingly, $\phi(t)$ is given by $A^{m} \psi^{(m)}(t, \tau)=A^{m} d^{m} \psi(t, \tau) / d t^{m} ; t \in \mathbf{R}$. Consequently, the average DC power of the received IR-UWB waveform is expressed as follows:

$$
\bar{P}_{D C}=\frac{1}{R_{L}^{\prime}} \int_{-\infty}^{+\infty}\left|A^{m} \Psi^{(m)}(j \omega, \tau)\right|^{2} d \omega
$$

where $A^{m}$ is the amplitude of the un-modulated $m^{t h}$ order IR-UWB waveform $R_{L}^{\prime}=T_{s} R_{L}\left(\sum_{q=0}^{M-1} \sum_{p=1}^{P} p_{q} w_{p}^{2}\left|s_{j}^{q}\right|^{2}\right)^{-1}$ is the effective load resistance, $\Psi^{(m)}(j \omega, \tau)=\Im\left\{\psi^{(m)}(t, \tau)\right\}$ is the Fourier transform of $\psi^{(m)}(t, \tau)$, $\omega$ is the angular frequency and $\Im\left\{\right.$.\} denotes the Fourier transform operation. For simplicity, $R_{L}^{\prime}=1$ to ensure the generality of the conducted analysis and its independence of the particular system design specifications.

\section{UWB-TO-DC ENERGY HARVESTING UNDER THE FCC SPECTRAL CONSTRAINTS}

An IR-UWB signal is subject to power spectral density (PSD) constraints, denoted by $S_{F C C}(\omega)$, imposed by the FCC on its effective isotropic radiated power (EIRP) [12]. Therefore, to maximize the DC power extracted from an IR-UWB signal, is to maximize its peak PSD by adapting $A^{m}$ to $\max \left\{S_{F C C}(\omega)\right\}$. The FCC-compliant Fourier transform of the $m^{\text {th }}$ order Gaussian-based derivative is given by:

$$
\Psi_{n, F C C}^{(m)}(j \omega, \tau)=A_{g}^{m}(j \omega)^{m} \tau_{g} \sqrt{\pi} \exp \left(-\left(\omega \tau_{g}\right)^{2} / 2\right)
$$

where $n$ denotes a normalized version of $\Psi^{(m)}(j \omega, \tau), A_{g}^{m}$ is the normalization constant of the $m^{t h}$ order derivative of a Gaussian basis function. Likewise, the FCC-compliant Fourier transform of the $m^{\text {th }}$ order sech-based derivative is given by:

$$
\Psi_{n, F C C}^{(m)}(j \omega, \tau)=A_{s}^{m}(j \omega)^{m} 4 \pi \tau_{s} \operatorname{sech}\left(2 \pi \omega \tau_{s}\right)
$$

where $A_{s}^{m}$ is the normalization constant of the $m^{\text {th }}$ order derivative of a sech-based IR-UWB waveform. Throughout the following analysis, $\Psi_{n, F C C}^{(m)}(j \omega, \tau)$ is given by either the definitions in (6) or (7) as required. It is important to highlight that, the values of $A_{g}^{m}$ and $A_{s}^{m}$ are defined in terms of the global maximum angular frequencies of (8) and (9), respectively (designated by $\omega_{p, g}$ and $\omega_{p, s}$ respectively). 
Figures 2(a) and (b) plot the simulated RF spectra of Gaussian-based and sech-based IR-UWB waveforms, respectively, based on (6) and (7) along with the FCC spectral constraints. A closer look at this figure reveals that, it is reasonable to employ IR-UWB signaling waveforms who's PSDs are mostly concentrated in the 3.1-10.6 GHz band, where most of the EIRP of a UWB signal is permitted. Consequently, the average received power in (5) is approximated as follows:

$$
\bar{P}_{D C}=\int_{-\infty}^{+\infty}\left|\Psi_{n, F C C}^{(m)}(j \omega, \tau)\right|^{2} d \omega \approx 2 \int_{\omega_{L}}^{\omega_{H}}\left|\Psi_{n, F C C}^{(m)}(j \omega, \tau)\right|^{2} d \omega
$$

where $\omega_{L}=2 \pi f_{L}, \omega_{H}=2 \pi f_{H}$, and $f_{L}$ and $f_{H}$ are the lower and the upper frequencies that determine the bandwidth over which the maximum FCC PSD is allowed. The DC power extracted from a Gaussian-based IR-UWB waveform at the LPF output is analytically obtained by substituting (6) in (8) as follows:

$$
\bar{P}_{D C, g}\left(\tau_{g}\right)=G_{p} \times\left(\Xi\left(\omega_{H} \tau_{g} / \sqrt{2}\right)-\Xi\left(\omega_{L} \tau_{g} / \sqrt{2}\right)\right)
$$

where $G_{p}=2 \pi\left(\tau_{g} A_{g}^{m}\right)^{2}\left(\sqrt{2} / \tau_{g}\right)^{m+1}$ and $\Xi(u)$ is given by:

$$
\Xi(u)=(1-s) \Gamma\left(\frac{m+s+1}{2}\right)(1-2 Q(\sqrt{2} u))-\exp \left(-u^{2}\right) \sum_{n=0}^{N-1} \frac{\Gamma\left(\frac{m+1}{2}\right)}{\Gamma\left(\frac{m+1}{2}-n\right)} u^{m-2 n-1}
$$

with $s=(m+1) \bmod 2, N=(m+s) / 2, \Gamma(z)$ and $Q(u)$ are the conventional Gamma function and the Gaussian $Q$-function, respectively. Similarly, the average received DC power of a sech-based IR-UWB waveform is obtained analytically by substituting (7) in (8) as follows:

$$
\bar{P}_{D C, g}\left(\tau_{g}\right)=S_{p} \times\left(\Lambda\left(2 \pi \omega_{H} \tau_{s}\right)-\Lambda\left(2 \pi \omega_{L} \tau_{s}\right)\right)
$$

where $S_{p}=8 m !\left(4 \pi \tau_{s} A_{s}^{m}\right)^{2}\left(1 / 2 \pi \tau_{s}\right)^{m+1}$, and $\Lambda(u)$ is given by:

$$
\Lambda(u)=\sum_{i=0}^{I} \sum_{a=0}^{m} \frac{(-1)^{a+i}(i+1)}{(m-a) !(-2(i+1))^{a+1}} \times u^{m-a} \exp (-2(i+1) u) ; I \gg 1
$$

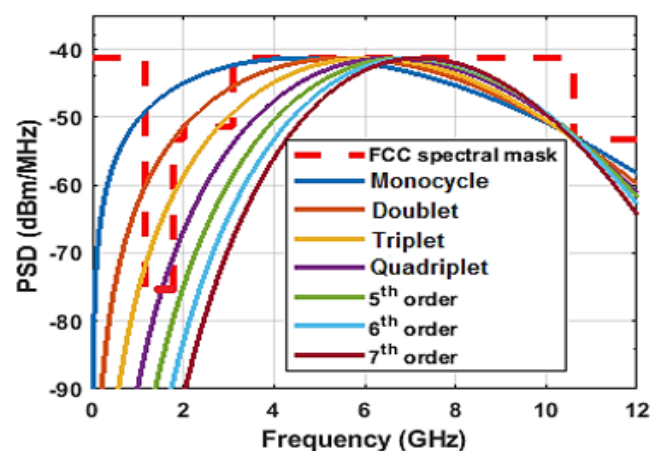

(a)

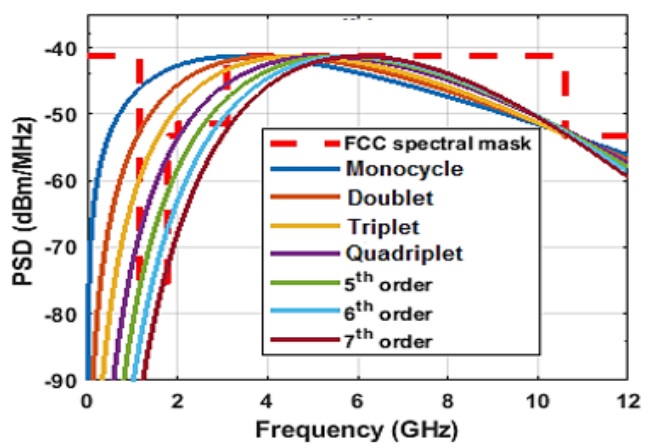

(b)

Figure 2. Spectra of (a) Gaussian-based IR-UWB waveforms and (b) sech-based IR-UWB waveforms

\section{SIMULATION RESULTS AND ANALYSIS}

In this section, the analytical expressions for the average received DC power predicted by (9) and (11) are evaluated. Furthermore, the obtained results are compared to the results obtained by system-level numerical simulations using MATLAB. For each basis functions, the FWHM pulse width, $\tau$, is changed from $1 \mathrm{ps}$, up to $500 \mathrm{ps}$ for the Gaussian basis function profile, and up to $250 \mathrm{ps}$ for the sech basis function. A 
maximum PSD of $\max \left\{S_{F C C}(\omega)\right\}=-41.3 \mathrm{dBm} / \mathrm{MHz}$ is assumed over a huge bandwidth from $f_{L}=3.1 \mathrm{GHz}$ $f_{H}=10.6 \mathrm{GHz}$ [12]. Throughout simulations, on-off keying (OOK) modulation is considered. The value of the relative time shift $\mu_{j}^{q}$ is set to zero. A pseudo-random binary sequence (PRBS) of $2^{31}-1$ bits length is generated in MATLAB. Each of the generated symbols is encoded by an IR-UWB waveform using the values of the afore-mentioned parameters. For each pulse width, the duration of the waveform (symbol) is defined over a symbol duration that includes, at least, $90 \%$ of the waveform energy. A number of 100 realizations of the channel impulse response is generated. For each realization, the power of the received signal is evaluated. The calculated DC power levels are averaged over the number of realizations of the channel impulse response.

Figure 3(a) shows the variations of the average received DC powers of Gaussian-based IR-UWB waveforms with $\tau$ for $m=1$ to 7 . Considering a particular value of $m$, the average received DC power increases monotonically from $0 \mu \mathrm{W}$, up to a global maximum value, which is obtained at an optimum value $T$. Further increase of $\tau$ beyond this optimum value reduces the average received DC power below this maximum value. For instance, maximum DC power of $0.29 \mu \mathrm{W}$ can be extracted from an FCC compliant Gaussian monocycle waveform, derived from a Gaussian basis function that have a FWHM pulse width of 50.51 ps. Although higher order derivative waveforms, such as doublets and triplets, show similar behaviors, the value of the optimum FWHM pulse width and the corresponding maximum DC power level extractable from each waveform decreases with increasing the derivative order. Increasing the derivative order $m$ leads to increasing the bandpass characteristics of the IR-UWB signal through the partial migration of its PSD to higher frequencies outside this spectral window. As a consequence, lower DC power levels are observed at the Rx output through this window.

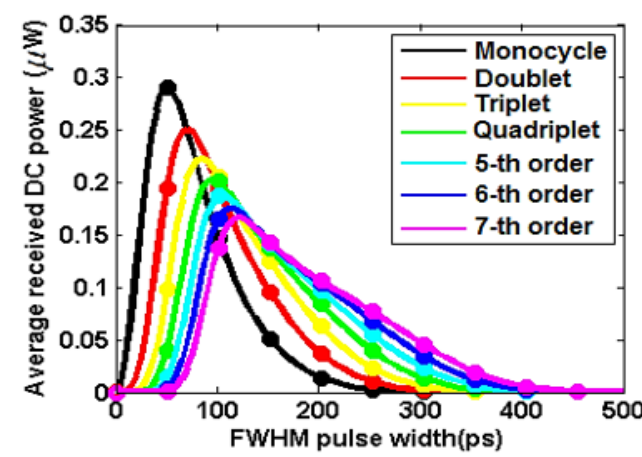

(a)

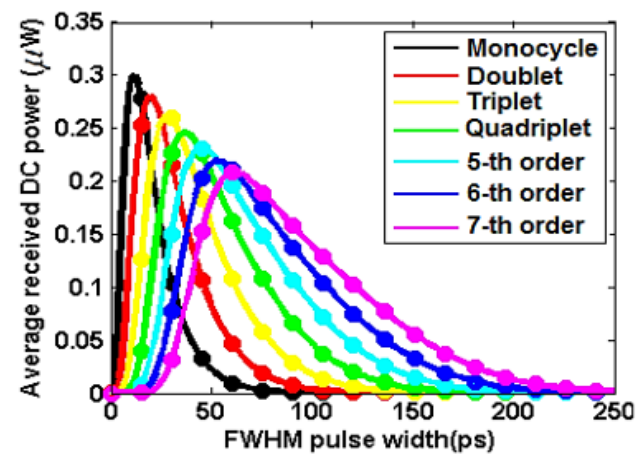

(b)

Figure 3. The average received DC powers versus the FWHM pulse width of (a) Gaussian-based IR-UWB waveforms and (b) sech-based IR-UWB waveforms

On the other hand, Figure 3(b) presents the variation of the average DC power versus $\tau$ for IRUWB waveforms, derived from a sech basis function. Likewise, the sech-based monocycle pulse achieves a maximum DC power level of $0.3 \mu \mathrm{W}$ at a FWHM input pulse width of 12.07 ps. Moreover, the FCCcompliant higher order derivatives of a sech basis function show similar behaviours to their Gaussian-based counterparts, in terms of the global maximum DC power levels achieved at optimum FWHM pulse widths. Both Gaussian and sech-based monocycles outperform their corresponding higher order derivatives in terms of the maximum extractable DC powers. These observations recommend IR-UWB waveforms for energy harvesting applications that need sub- $\mu$ Watt power levels [15].

\section{POTENTIAL APPLICATION SCENARIOS}

It should be highlited that, the particular application scenario to which an $\mathrm{EH}$ is integrable depends strongly on the power levels achievable from this EH. Table 1 [16] the lists a number of power resources from, including the UWB spectrum. Obviously, the power level expected from an IR-UWB EH is comparable to the range expected from an RF signal emitted by a building-top basestation employed for mobile services. This competitive performance is attributed to the huge bandwidth dedicated to these signals, which compensates the extremely low PSD limits imposed to UWB signals. Such kind of $\mu$-power converters is highly recommended for microelectronic mechanical systems (MEMS) relying on the CMOS technology. 
Table 1. Comparison of the available energy resources [16]

\begin{tabular}{|c|c|c|}
\hline Energy Source & Emitted Power & Harvested Power \\
\hline \multicolumn{3}{|l|}{ Ambient Light } \\
\hline Indoor & $0.1 \mathrm{~mW} / \mathrm{cm}^{2}$ & $10 \mu \mathrm{W} / \mathrm{cm}^{2}$ \\
\hline Outdoor & $100 \mathrm{~mW} / \mathrm{cm}^{2}$ & $10 \mathrm{~mW} / \mathrm{cm}^{2}$ \\
\hline \multicolumn{3}{|l|}{ Vibration/Motion } \\
\hline Human & $0.5 \mathrm{~m} @ 1 \mathrm{~Hz}$ & \\
\hline Human & $1 \mathrm{~m} / \mathrm{s}^{2} @ 50 \mathrm{kHz}$ & $4 \mu \mathrm{W} / \mathrm{cm}^{2}$ \\
\hline Industry & $1 \mathrm{~m} @ 5 \mathrm{~Hz}$ & \\
\hline Industry & $10 \mathrm{~m} / \mathrm{s}^{2} @ 5 \mathrm{kHz}$ & $100 \mu \mathrm{W} / \mathrm{cm}^{2}$ \\
\hline \multicolumn{3}{|l|}{ Thermal Energy } \\
\hline Human & $20 \mathrm{~mW} / \mathrm{cm}^{2}$ & $30 \mu \mathrm{W} / \mathrm{cm}^{2}$ \\
\hline Indistry & $100 \mathrm{~mW} / \mathrm{cm}^{2}$ & $1-10 \mathrm{~mW} / \mathrm{cm}^{2}$ \\
\hline $\begin{array}{c}\text { RF } \\
\text { GSM Basestation } \\
\text { This work }\end{array}$ & $0.3 \mu \mathrm{W} / \mathrm{cm}^{2}$ & $0.1 \mu \mathrm{W} / \mathrm{cm}^{2}$ \\
\hline RF-IR-UWB & $0.56 \mathrm{~mW}(3.1-10.6 \mathrm{GHz})$ & $0.3 \mu \mathrm{W}$ \\
\hline
\end{tabular}

Assuming a uniform plane wavefront incident to an $\mathrm{EH}$ antenna, the spatial power density at the EH side, denoted by $D$, is given by $D=\bar{P}_{D C} / A_{E H}$, where $A_{E H}$ is the effective radiation exposure area of the EH antenna. Considering a time average UWB signal power of $\overline{P_{D C}}=0.3 \mu \mathrm{W}$ as in Table 1 and an effective antenna area of $A_{E H}=1 \mathrm{~cm}^{2}$, results in a theoretically estimated spatial power density of $D=0.3 \mu \mathrm{W} / \mathrm{cm}^{2}$. The hypothesized antenna size is comparable to many of the state-of-the-art antenna designs published on UWB energy harvesting [17-25]. Moreover, it is clear that the theoretically expected value of $D=0.3 \mu \mathrm{W} / \mathrm{cm}^{2}$ is comparable to the values listed in Table 1 for RF EH techniques, which are based on much narrower frequency bands and much larger antenna dimensions, accordingly.

\section{CONCLUSION}

This paper investigates the feasibility of energy harvesting in the useful ultrawide band of the spectrum (i.e., 3.1-10.6 GHz). Analytical expressions for the maximum DC power levels that can be extracted from these waveforms are derived. Simulation results indicate that energy harvesting in this band finds its best applications where there is a need for $s u b-\mu W a t t$ power levels. These applications include, but are not limited to, micro-electro-mechanical systems (MEMS), especially robotic-based MEMS developed for in-body medical missions, where the accessibility to these nodes is essentially impossible after deployment. The reported analysis provides a good starting point for further theoretical and/or experimental investigations that consider specific realizations of ultrawide band communication/energy harvesting systems. Ongoing research on the realizability of UWB energy harvesters is being conducted in ordre to assess the feasibility of these techniqes for the recommended potential applications.

\section{REFERENCES}

[1] M. R. V. Moghadam, Y. Zeng and R. Zhang, "Waveform optimization for radio-frequency wireless power transfer: (Invited paper)," in 2017 IEEE 18th International Workshop on Signal Processing Advances in Wireless Communications (SPAWC), Sapporo, 2017, pp. 1-6.

[2] M. Alfaqawi, M. H. Habaebi, M. R. Islam and M. U. Siddiqi, "Energy Harvesting Network with Wireless Distributed Computing," in IEEE Systems Journal, vol. 13, no. 3, pp. 2605-2616, 2019.

[3] Y. Lu et al., "A Wide Input Range Dual-Path CMOS Rectifier for RF Energy Harvesting," in IEEE Transactions on Circuits and Systems II: Express Briefs, vol. 64, no. 2, pp. 166-170, 2017.

[4] T. A. Elwi, Z. Asaad, A. Hassain, and O. A. Tawfeeq, "Hilbert metamaterial printed antenna based on organic substrates for energy harvesting," IET Microwaves, Antennas \& Propagation, vol. 13, no. 12, pp. 2185-2192, 2019.

[5] F. Alneyadi, M. Alkaabi, S. Alketbi, S. Hajraf and R. Ramzan, "2.4GHz WLAN RF energy harvester for passive indoor sensor nodes," 2014 IEEE International Conference on Semiconductor Electronics (ICSE2014), Kuala Lumpur, 2014, pp. 471-474.

[6] S. Zheng, W. Liu and Y. Pan, "Design of an Ultra-Wideband High- Efficiency Rectifier for Wireless Power Transmission and Harvesting Applications," in IEEE Transactions on Industrial Informatics, vol. 15, no. 6, pp. 3334-3342, 2019.

[7] J. Kimionis, A. Collado, M. M. Tentzeris, and A. Georgiadis, "Octave and Decade Printed UWB Rectifiers Based on Nonuniform Transmission Lines for Energy Harvesting," IEEE Transactions on Microwave Theory and Techniques, vol. 65 , no. 11, pp. 4326-4334, 2017.

[8] T. A. Elwi, O. Almukhtar Tawfeeq, Y. Alnaiemy, H. S. Ahmed and N. Lajos, "A UWB Monopole Antenna Design based RF Energy Harvesting Technology," 2018 Third Scientific Conference of Electrical Engineering (SCEE), Baghdad, Iraq, 2018, pp. 111-115. 
[9] T. A. Elwi, "Novel UWB printed metamaterial microstrip antenna based organic substrates for RF-energy harvesting applications," AEU-International Journal of Electronics and Communications, vol. 101, pp. 44-53, 2019.

[10] Y. Zeng, B. Clerckx and R. Zhang, "Communications and Signals Design for Wireless Power Transmission," IEEE Transactions on Communications., vol. 65, no. 5, pp. 2264-2290, 2017.

[11] A. Collado and A. Georgiadis, "Optimal waveforms for efficient wireless power transmission," in IEEE Microwave and Wireless Components Letters, vol. 24, no. 5, pp. 354-356, 2014.

[12] US. Federal Communications Commission, First report and order, "Revision of part 15 of the commission's rules regarding ultra-wideband transmission systems," EDOCS, 2002.

[13] S. T. Abraha, et al., "Performance Evaluation of IR-UWB in Short-Range Fiber Communication Using Linear Combination of Monocycles," in Journal of Lightwave Technology, vol. 29, no. 8, pp. 1143-1151, 2011.

[14] A. Mehbodniya and S. Aissa, "Effects of MB-OFDM System Interference on the Performance of DS-UWB," in IEEE Transactions on Vehicular Technology, vol. 58, no. 8, pp. 4665-4669, 2009.

[15] J. Iannacci, "Microsystem based Energy Harvesting (EH-MEMS): Powering pervasivity of the Internet of Things (IoT)-A review with focus on mechanical vibrations," Journal of King Saud University-Science, vol. 31, no. 1, pp. 66-74, 2019.

[16] R. J. M. Vullers, R. v. Schaijk, H. J. Visser, J. Penders and C. V. Hoof, "Energy Harvesting for Autonomous Wireless Sensor Networks," in IEEE Solid-State Circuits Magazine, vol. 2, no. 2, pp. 29-38, 2010.

[17] H. Lyu, Z. Wang and A. Babakhani, "A UHF/UWB Hybrid RFID Tag With a 51-m Energy-Harvesting Sensitivity for Remote Vital-Sign Monitoring," in IEEE Transactions on Microwave Theory and Techniques, 2020.

[18] T. Haapala, T. Rantataro and K. A. I. Halonen, "A Fully Integrated Programmable 6.0-8.5GHz UWB IR Transmitter Front-End for Energy-Harvesting Devices," in IEEE Journal of Solid-State Circuits, vol. 55, no. 7, pp. 1922-1934, 2020.

[19] A. Quddious, M. A. B. Abbasi, M. A. Antoniades, P. Vryonides, V. Fusco and S. Nikolaou, "Dynamically Reconfigurable UWB Antenna Using an FET Switch Powered by Wireless RF Harvested Energy," in IEEE Transactions on Antennas and Propagation, vol. 68, no. 8, pp. 5872-5881, 2020.

[20] G. Srinivasu, N. Anveshkumar and V. K. Sharma, "A Compact Octagon Slotted Circular UWB Antenna for RF Energy Harvesting," 2020 International Conference on Emerging Trends in Information Technology and Engineering (ic-ETITE), Vellore, India, 2020, pp. 1-5.

[21] T. Gayatri, N. Anveshkumar and V. K. Sharma, "A Compact Planar UWB Antenna for Spectrum Sensing in Cognitive Radio," 2020 International Conference on Emerging Trends in Information Technology and Engineering (ic-ETITE), Vellore, India, 2020, pp. 1-5.

[22] T. Gayatri, N. Anveshkumar and V. K. Sharma, "A Hexagon Slotted Circular Monopole UWB Antenna for Cognitive Radio Applications," 2020 International Conference on Emerging Trends in Information Technology and Engineering (ic-ETITE), Vellore, India, 2020, pp. 1-5.

[23] G. Srinivasu, N. Anveshkumar and V. K. Sharma, "A Circular Slotted Planar Monopole UWB Antenna for RF Energy Harvesting Applications," 2020 International Conference on Emerging Trends in Information Technology and Engineering (ic-ETITE), Vellore, India, 2020, pp. 1-5.

[24] S. Schmickl, T. Faseth and H. Pretl, "A 1.9- $\mu$ W 7-GHz IR-UWB Transmitter with RF-Energy-Harvester in 180-nm CMOS for Battery-Less Bio-Sensors," 2019 17th IEEE International New Circuits and Systems Conference (NEWCAS), Munich, Germany, 2019, pp. 1-4.

[25] T. A. Elwi, O. Almukhtar Tawfeeq, Y. Alnaiemy, H. S. Ahmed and N. Lajos, "A UWB Monopole Antenna Design based RF Energy Harvesting Technology," 2018 Third Scientific Conference of Electrical Engineering (SCEE), Baghdad, Iraq, 2018, pp. 111-115.

\section{BIOGRAPHIES OF AUTHORS}

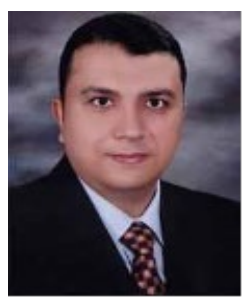

Nasr Rashid was born in Egypt, in 1973. He received the B.Sc. (with highest honors), M.Sc., and $\mathrm{PhD}$ in Electronics and Communication Engineering from Al-Azhar University, Cairo, Egypt in 1996, 2004, and 2009 respectively. He is currently an Assistant Professor in the Department of Electrical Engineering, College of Engineering, Jouf University, Sakaka, Kingdom of Saudi Arabia (KSA). His current research interests are in control systems, power electronics, wirless communations and energy harvesting.

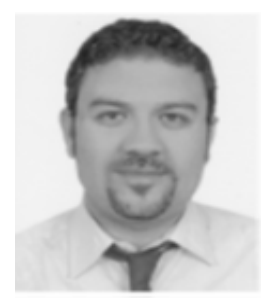

Mohamed Shehata received the B.Sc. and M.Sc. degrees in Electronics and Communications Engineering from Al-Azhar University, Cairo, Egypt, in 2006 and 2012, respectively. Currently, he is working with the Department of Research Training, Scientific Outcomes of Undergraduate Research Students (SOURCE), Egypt. His current research interests include, but are not limited to; wireless communications, antenna theory and techniques, and microwave photonics with a particular focus radio over fiber enabling technologies for $5 \mathrm{G}$ communications. 